\title{
Dry Eye Disease Is Associated with Deterioration of Mental Health in Male Japanese University Staff
}

\author{
Kaoru Tounaka, ${ }^{1,4, *}$ Kenya Yuki, ${ }^{2, *}$ Keisuke Kouyama, ${ }^{3}$ Takayuki Abe, ${ }^{3}$ \\ Kazuo Tsubota, ${ }^{2}$ Hiroshi Kawabe ${ }^{1}$ and Kazuhito Yokoyama ${ }^{4}$ \\ ${ }^{1}$ Health Center, Keio University, Fujisawa, Kanagawa, Japan \\ ${ }^{2}$ Department of Ophthalmology, Keio University School of Medicine, Tokyo, Japan \\ ${ }^{3}$ The Center for Clinical Research, Keio University School of Medicine, Tokyo, Japan \\ ${ }^{4}$ Department of Epidemiology and Environmental Health, Juntendo University Faculty of Medicine, Tokyo, Japan
}

Dry eye disease (DED) is defined as a multifactorial disease of the tears and ocular surface that results in symptoms of discomfort, visual disturbance, and tear-film instability, with potential damage to the ocular surface. It is thought to be associated with reduced quality of life (QOL). The aim of the present study was to investigate the effects of DED on health-related QOL in Japanese university sedentary office workers who are daily users of visual display terminal. In this study, 163 university staff (99 male and 64 female), aged 23-69 years, served as study subjects. Subjects were asked to answer the following three questions. (1) How often do your eyes feel dry? (2) How often do your eyes feel irritated? (3) Have you ever been diagnosed by a clinician as having dry eye syndrome? Sixty-eight subjects who answered "constantly," "often", or "sometimes" to both questions 1 and 2 were classified as the DED Group, and the remaining 95 were defined as the Non-DED Group. QOL was assessed by the SF-36 questionnaire, which consisted of 36 items to produce three summary scores, namely, mental, physical, and role/social component summary scores. For males, the DED Group had significantly lower scores than the Non-DED Group for mental component summary (MCS) $(P=0.005)$. In multiple regression analysis, MCS scores were adversely related to DED in males $(P=0.015)$. DED was associated with worsened QOL. DED should be regarded as a factor that can lead to deterioration of mental health.

Keywords: dry eye disease; gender difference; mental health; quality of life; SF-36

Tohoku J. Exp. Med., 2014 July, 233 (3), 215-220. (C) 2014 Tohoku University Medical Press

\section{Introduction}

Dry eye disease (DED) is defined as a multifactorial disease of the tears and ocular surface that results in symptoms of discomfort, visual disturbance, and tear-film instability, with potential damage to the ocular surface. It is accompanied by increased osmolality of the tear film and inflammation of the ocular surface (Lemp et al. 2007). DED is one of the most common ocular surface diseases worldwide. The prevalence of DED has been reported to be from $\sim 4.4 \%$ to as high as $50 \%$ among middle-aged and elderly people (Schein et al. 1997; McCarty et al. 1998; Nichols et al. 2002; Schaumberg et al. 2003; Moss et al. 2008; Guo et al. 2010; Galor et al. 2011). DED causes constant irritation, ocular discomfort, fatigue, and visual disturbance, which interfere with daily activities (Miljanovic et al. 2007), disturbing one's ability to perform various tasks that require sustained visual concentration. According to a survey in clinic-based populations, quality of life (QOL), including mental health, was significantly lower in patients with severe DED than in the control group (Mertzanis et al. 2005; Le et al. 2012; Li et al. 2012; Sakane et al. 2013). The decrease in QOL caused by DED is an important societal health issue, because this condition results in a larger burden on society through costs of care and treatment, as well as those associated with the disease itself (Lemp et al. 2007).

In Japan, a large population-based study revealed that the prevalence of clinically diagnosed DED was $2.1 \%$ and $7.9 \%$ in males and females, respectively, and the percentages with severe symptoms were $11.5 \%$ and $18.7 \%$ in males and females, respectively (Uchino et al. 2011). However, to the best of our knowledge, no study has ever examined the relationship between DED and QOL in non-clinic-based subjects in Japan, whereas DED has been reported to be related to poor QOL and depression in a general population

Received April 1, 2014; revised and accepted June 16, 2014. Published online July 23, 2014; doi: 10.1620/tjem.233.215.

*These two authors contributed equally to this work.

Correspondence: Kazuhito Yokoyama, M.D., D.M.Sc., Department of Epidemiology and Environmental Health, Juntendo University

Faculty of Medicine, 2-1-1 Hongo, Bunkyo-ku, Tokyo 113-8421, Japan.

e-mail: kyokoya@juntendo.ac.jp 
in other countries (Galor et al. 2011; Le et al. 2012; Wang et al. 2012). The major purpose of the present study is, therefore, clarify the relationship between DED and QOL in non-clinic-based Japanese subjects.

The impact of DED on QOL has been evaluated by several questionnaires (Nichols et al. 2002; Mertzanis et al. 2005; Kim et al. 2011; Le et al. 2012; Li et al. 2012; Sakane et al. 2013). For example, the visual function questionnaire such as the National Eye Institute's Visual Function Questionnaire 25 (NEI VFQ-25) has been used in many studies (Nichols et al. 2002; Le et al. 2012; Li et al. 2012). However, few studies have used the 36-Item Short Form Health Survey (SF-36) questionnaire, which is a multi-purpose questionnaire that evaluates mental, physical, and social aspects of QOL (Mertzanis et al. 2005), and is considered useful in surveys of general and specific populations, comparing the relative burden between various diseases, and in assessing the health benefits of a specific method of medical treatment (Fukuhara et al. 1998a, b; Fukuhara and Suzukamo 2004). SF-36 is capable of evaluating QOL from three aspects by calculating mental, physical, and role/social component summary (MCS, PCS, and RCS) scores from the eight subscale scores based on factor analysis (Fukuhara and Suzukamo 2004). Thus, SF-36 was used in the present study for the assessment of QOL.

\section{Methods}

Study Subjects

A total of 361 university staff at the Shonan Fujisawa Campus of Keio University were asked to participate in the study during their annual health checkups in November 2011. All the subjects in this study were sedentary office workers who used a visual display terminal (VDT) for their work. Eight were excluded because the logarithm of the minimum angle of resolution of corrected or uncorrected visual acuity was $<0.16$, or they were being treated for glaucoma, diabetic retinopathy, or any other systemic diseases that might have affected ocular surface condition, such as Sjögren's syndrome. Of the 355 eligible candidates, 190 agreed to participate in the study. No significant differences were observed between the 190 participants and the remaining 165 persons in terms of age, sex, or uncorrected/corrected visual acuity $(P>0.05)$. All 190 subjects received ophthalmic examinations by a single ophthalmologist (Kenya Yuki). The examination included corrected visual acuity measurements, slit-lamp biomicroscopy with fluorescein staining (only for non-contact lens users), and funduscopy without mydriasis by direct ophthalmoscopy.

The study was approved by the Ethics Review Subcommittee of the Keio University Research Ethics Committee, and was pre-registered in the UMIN Clinical Trial Registry (UMIN6271, http://www. umin.ac.jp/ctr/index.htm). The procedures used in this study conformed to the tenets of the Declaration of Helsinki. The nature of the procedure was explained to all the participants, and the study was conducted with their written informed consent. The study was carried out during November 7-11, 2011 at the Keio University Shonan Fujisawa Campus, Fujisawa, Kanagawa, Japan.

\section{Questionnaire}

All 190 subjects were asked to answer the following three ques- tions developed and validated by Schaumberg and coworkers for the screening of DED (Schaumberg et al. 2001, 2003; Gulati et al. 2006):

(1) How often do your eyes feel dry?

(2) How often do your eyes feel irritated?

(3) Have you ever been diagnosed by a clinician as having dry eye syndrome?

The response options for the first two questions were "constantly," "often", "sometimes", or "never", and for the third question, "yes" or "no." The questions and response options were translated into Japanese from the original English, and verified by back-translation into English and comparison with the original (Uchino et al. 2011). Twenty-seven participants who responded "yes" to the third question were excluded from the analysis, because their QOL might have been affected by being diagnosed with DED.

Sixty-eight subjects who answered "constantly", "often", or "sometimes" to both questions 1 and 2 were classified as the DED Group, and the remaining 95 were defined as the Non-DED Group.

QOL was assessed by the Japanese version of SF-36, which consists of 36 items, for which the reliability and validity have been reported with normative data, and it is considered applicable to those aged 20-70 years (Fukuhara et al. 1998a, b; Fukuhara and Suzukamo 2004). Subjects were asked to answer each item on the following eight scales by 3-6 levels of response during the past 4 weeks: physical functioning, role-physical, bodily pain, general health perception, vitality, social functioning, role-emotional, and mental health. MCS, PCS, and RCS scores were then calculated from these eight scale scores according to the manual.

A self-administered questionnaire was used to obtain the following characteristics: subjective quality of vision, method for correcting visual acuity, smoking habit, exercise well, and history of hypertension, diabetes mellitus, hyperlipidemia, or mental disease and treatment.

\section{Statistical Analysis}

Differences in variables between the DED and Non-DED Groups were examined by the Mann-Whitney $U$-test, Fisher's exact probability test, or $\chi^{2}$ test. Stepwise multiple linear regression analysis was performed to explore dependent variables significantly related to SF-36 summary scores. In this analysis, age, DED or Non-DED, contact lenses use, smoking habit, exercise well, and subjective quality of vision were entered and removed as dependent variables into the regression equation with a significance level of $P<0.05$. All analyses were conducted using SPSS version 20.0 statistical package (SPSS, Chicago, IL, USA).

\section{Results}

The characteristics of the 163 subjects by sex are shown in Table 1. The DED Group accounted for $41.7 \%$ of subjects. The average age in males was significantly higher than that in females. The proportion of contact lens users was significantly higher in females than males. No significant differences were observed in subjective quality of vision, smoking habit, exercise well, or the rates of hypertension, diabetes, hyperlipidemia, or mental disease between the sexes. The characteristics between the DED and Non-DED Groups according to sex are shown in Table 2. The proportion of female contact lens users was significantly higher in the DED than Non-DED Group. No signif- 
Table 1. Differences in Characteristics between 99 Male and 64 Female Subjects: Average \pm Standard Deviation or Number $(\%)$.

\begin{tabular}{|c|c|c|c|}
\hline & Male subjects & Female subjects & $P$ value \\
\hline Age (years) & $46 \pm 11$ & $41 \pm 10$ & $0.024 *$ \\
\hline DED Group & $36(36)$ & $32(50)$ & $0.104^{\dagger}$ \\
\hline Non-DED Group & $63(64)$ & $32(50)$ & \\
\hline \multicolumn{4}{|c|}{ Subjective quality of vision } \\
\hline Fine & $79(80)$ & $53(83)$ & $0.687^{\dagger}$ \\
\hline Not fine & $20(20)$ & $11(17)$ & \\
\hline \multicolumn{4}{|c|}{ Method for correcting visual acuity } \\
\hline Contact lenses use & $11(11)$ & $18(28)$ & $0.001^{*}$ \\
\hline Glasses use & $50(51)$ & $15(24)$ & \\
\hline Naked eye & $38(38)$ & $31(48)$ & \\
\hline \multicolumn{4}{|l|}{ Smoking habit } \\
\hline Yes & $6(6)$ & $0(0)$ & $0.082^{\dagger}$ \\
\hline No & $93(94)$ & $64(100)$ & \\
\hline \multicolumn{4}{|l|}{ Exercise well } \\
\hline Yes & $32(32)$ & $16(25)$ & $0.380^{\dagger}$ \\
\hline No & $67(68)$ & $48(75)$ & \\
\hline \multicolumn{4}{|l|}{ Hypertension } \\
\hline Yes & $10(10)$ & $4(6)$ & $0.569^{\dagger}$ \\
\hline No & $89(90)$ & $60(94)$ & \\
\hline \multicolumn{4}{|l|}{ Diabetes } \\
\hline Yes & $3(3)$ & $0(0)$ & $0.280^{\dagger}$ \\
\hline No & $96(97)$ & $64(100)$ & \\
\hline \multicolumn{4}{|l|}{ Hyperlipidemia } \\
\hline Yes & $7(7)$ & $1(2)$ & $0.150^{\dagger}$ \\
\hline No & $92(93)$ & $63(98)$ & \\
\hline \multicolumn{4}{|l|}{ Mental disease } \\
\hline Yes & $1(1)$ & $1(2)$ & $1.000^{\dagger}$ \\
\hline No & 98 (99) & $63(98)$ & \\
\hline
\end{tabular}

*Mann-Whitney $U$-test, "Fisher's exact probability test, ${ }^{\star} \chi^{2}$ test.

The average age in males was significantly higher than that in females.

icant differences were observed in subjective quality of vision, smoking habit, exercise, or the rates of hypertension, diabetes, hyperlipidemia, or mental disease between the DED and Non-DED Groups.

SF-36 scores for the DED and Non-DED Groups are shown in Table 3. For males, the DED Group had a significantly lower score than the Non-DED Group for MCS score. For both sexes, the DED Group had an average MCS below the Japanese norm of 50 (Fukuhara et al. 1998a, b; Fukuhara and Suzukamo 2004). Table 4 shows the results of a stepwise multiple linear regression analysis. DED was significantly related to MCS score in males. In contrast, DED was not significantly related to PCS and RCS scores in either males or females $(P>0.05)$.

\section{Discussion}

For males, the DED Group had significantly lower MCS scores than the Non-DED Group had. This agrees with previous studies in which DED had a negative effect on mental health (Mertzanis et al. 2005; Galor et al. 2011; Kim et al. 2011; Le et al. 2012; Li et al. 2012; Wang et al. 2012; Sakane et al. 2013). The study on a non-clinic-based population in China showed lower QOL for mental health in subjects with DED (Le et al. 2012). In a survey of the general population aged $\geq 65$ years in Korea, the DED Group had a higher rate of depression compared with the Non-DED Group (Kim et al. 2011). Wang et al. (2012) demonstrated significantly higher prevalence of comorbidity in patients with depression and DED in Taiwan. Thus, it seems that even a small degree of ocular discomfort or loss of vision due to DED significantly affects mental health in the general population. However, patients with depression might be more prone to experience dry eye symptoms, therefore, the negative mental condition may possibly enhance complaints of dry eye symptoms in the general population (Galor et al. 2011; Kim et al. 2011; Wang et al. 
Table 2. The Characteristics between the DED and Non-DED Groups according to Sex Subjects: Average \pm Standard Deviation or Number $(\%)$.

\begin{tabular}{|c|c|c|c|c|c|c|}
\hline & \multicolumn{3}{|c|}{ Male subjects } & \multicolumn{3}{|c|}{ Female subjects } \\
\hline & $\begin{array}{c}\text { DED } \\
(n=36)\end{array}$ & $\begin{array}{l}\text { Non-DED } \\
(n=63)\end{array}$ & $P$ value & $\begin{array}{c}\text { DED } \\
(n=32)\end{array}$ & $\begin{array}{l}\text { Non-DED } \\
(n=32)\end{array}$ & $P$ value \\
\hline Age (yrs) & $42 \pm 11$ & $48 \pm 11$ & $0.019^{*}$ & $38 \pm 10$ & $45 \pm 9$ & $0.006^{*}$ \\
\hline \multicolumn{7}{|c|}{ Subjective quality of vision } \\
\hline Fine & $28(78)$ & $51(81)$ & $0.796^{\dagger}$ & $27(84)$ & $26(81)$ & $1.000^{\dagger}$ \\
\hline Not fine & $8(22)$ & $12(19)$ & & $5(16)$ & $6(19)$ & \\
\hline \multicolumn{7}{|c|}{ Method for correcting visual acuity } \\
\hline Contact lenses & $6(17)$ & $5(8)$ & $0.413^{\ddagger}$ & $14(44)$ & $4(12)$ & $0.003^{*}$ \\
\hline Spectacles & $17(47)$ & $33(52)$ & & $9(28)$ & $6(19)$ & \\
\hline Naked eye & $13(36)$ & $25(40)$ & & $9(28)$ & $22(69)$ & \\
\hline \multicolumn{7}{|l|}{ Smoking habit } \\
\hline Yes & $1(3)$ & $5(8)$ & $0.412^{\dagger}$ & $0(0)$ & $0(0)$ & - \\
\hline No & $35(97)$ & $58(92)$ & & $32(100)$ & $32(100)$ & \\
\hline \multicolumn{7}{|l|}{ Exercise well } \\
\hline Yes & $9(25)$ & $23(37)$ & $0.271^{\dagger}$ & $5(16)$ & $11(34)$ & $0.148^{\dagger}$ \\
\hline No & $27(75)$ & $40(63)$ & & $27(84)$ & $21(66)$ & \\
\hline \multicolumn{7}{|l|}{ Hypertension } \\
\hline Yes & $3(8)$ & $7(11)$ & $0.743^{\dagger}$ & $3(9)$ & $1(3)$ & $0.613^{\dagger}$ \\
\hline No & $33(92)$ & $56(89)$ & & $29(91)$ & $31(97)$ & \\
\hline \multicolumn{7}{|l|}{ Diabetes } \\
\hline Yes & $0(0)$ & $3(5)$ & $0.552^{\dagger}$ & $0(0)$ & $0(0)$ & - \\
\hline No & $36(100)$ & $60(95)$ & & $32(100)$ & $32(100)$ & \\
\hline \multicolumn{7}{|l|}{ Hyperlipidemia } \\
\hline Yes & $1(3)$ & $6(10)$ & $0.419^{\dagger}$ & $1(3)$ & $0(0)$ & $1.000^{\dagger}$ \\
\hline No & $35(97)$ & $57(90)$ & & $31(97)$ & $32(100)$ & \\
\hline \multicolumn{7}{|l|}{ Mental disease } \\
\hline Yes & $0(0)$ & $1(2)$ & $1.000^{\dagger}$ & $1(3)$ & $0(0)$ & $1.000^{\dagger}$ \\
\hline No & $36(100)$ & $62(98)$ & & $31(97)$ & $32(100)$ & \\
\hline
\end{tabular}

*Mann-Whitney $U$ test, ${ }^{\star F}$ Fisher’s exact probability test, ${ }^{\star} \chi^{2}$ test.

Table 3. Differences in Three Summary Scores of SF-36 between DED and Non-DED Groups by Sex*.

\begin{tabular}{|c|c|c|c|c|c|c|}
\hline \multirow{2}{*}{ Summary scores } & \multicolumn{3}{|c|}{ Male subjects } & \multicolumn{3}{|c|}{ Female subjects } \\
\hline & $\begin{array}{c}\text { DED } \\
(n=36)\end{array}$ & $\begin{array}{l}\text { Non-DED } \\
(n=63)\end{array}$ & $P$ value & $\begin{array}{c}\text { DED } \\
(n=32)\end{array}$ & $\begin{array}{l}\text { Non-DED } \\
(n=32)\end{array}$ & $P$ value \\
\hline Mental component & $48.2 \pm 10.0$ & $52.9 \pm 8.7$ & 0.005 & $47.0 \pm 8.5$ & $50.2 \pm 9.5$ & 0.101 \\
\hline Physical component & $56.2 \pm 7.2$ & $54.4 \pm 6.7$ & 0.118 & $54.2 \pm 6.9$ & $52.7 \pm 8.8$ & 0.667 \\
\hline Role/social component & $52.0 \pm 6.9$ & $52.3 \pm 7.0$ & 0.341 & $53.9 \pm 6.6$ & $52.4 \pm 8.0$ & 0.428 \\
\hline
\end{tabular}

*Values are average \pm standard deviation.

2012). Further studies are necessary to confirm our present findings.

In this study, the average age in the male group was significant higher than that in the female group. The difference in age may affect the difference in MCS score between the male and female groups. It is possible that duration of exposure to DED syndrome in male subjects was longer than in female subjects, which may have reduced MCS score in male DED subjects. However, as shown in Table 4, after multivariable adjustment, MCS score was associated with DED, but not age in male subjects. This suggests that the effect of difference in age between the male and female groups on MCS score is not strong.

In the present study, although QOL scores for mental 
Table 4. Variables Significantly Related to Three Summary Scores of SF-36 in 99 Males and 64 Females: Results of A Stepwise Multiple Regression Analysis*.

\begin{tabular}{|c|c|c|c|c|}
\hline \multirow[b]{2}{*}{ Dependent variable } & \multicolumn{2}{|r|}{ Male subjects } & \multicolumn{2}{|r|}{ Female subjects } \\
\hline & $\mathrm{R}^{2 \dagger}$ & $\begin{array}{c}\text { Variables selected } \\
\text { (standardized regression } \\
\text { coefficient) })^{P \text { value }}\end{array}$ & $\mathrm{R}^{2 \dagger}$ & $\begin{array}{c}\text { Variables selected } \\
\text { (standardized regression } \\
\text { coefficient) })^{P \text { value }}\end{array}$ \\
\hline Mental component & 0.049 & $\operatorname{DED}(-0.243)^{0.015}$ & 0.100 & Exercise well $(0.339)^{0.006}$ \\
\hline Physical component & 0.076 & Age $(-0.292)^{0.003}$ & 0.095 & Contact lenses use $(0.331)^{0.008}$ \\
\hline Role/social component & - & - & - & - \\
\hline
\end{tabular}

*Independent variables were age, DED or Non-DED, contact lenses use, smoking habit, exercise well, and subjective quality of vision.

${ }^{\dagger} \mathrm{R}^{2}=$ Multiple correlation coefficient adjusted for the degree of freedom.

Table 5. Differences in Three Summary Scores of SF-36 between DED and Non-DED Groups*.

\begin{tabular}{lccc}
\hline \multicolumn{1}{c}{ Summary scores } & $\begin{array}{c}\text { DED } \\
(n=68)\end{array}$ & $\begin{array}{c}\text { Non-DED } \\
(n=95)\end{array}$ & $P$ value \\
\hline Mental component & $47.6 \pm 9.3$ & $52.0 \pm 9.0$ & 0.001 \\
Physical component & $55.3 \pm 7.0$ & $53.8 \pm 7.4$ & 0.169 \\
Role/Social component & $52.5 \pm 7.1$ & $52.3 \pm 7.3$ & 0.946 \\
\hline
\end{tabular}

*Values are average \pm standard deviation.

health were lower than the reference values in Japan, no significant changes in QOL were observed between females in the DED and Non-DED Groups. Among 27 subjects with clinically diagnosed DED, who were excluded from the analysis, only five were men, therefore, a lower number of men with DED might have received medical treatment as compared with women. Thus, it is possible that, in the present study, the DED Group had more potent patients among males than females.

We observed no significant differences in PCS and RCS scores between the DED and Non-DED Groups. However, Mertzanis et al. (2005) reported that patients with serious DED consistently had lower role-physical and bodily pain scores, which were related to PCS and RCS scores, than the Non-DED Group using SF-36. The reason for the discrepancy is unclear. One possible explanation is the racial difference. Eighty-two percent of subjects in the study of Mertzanis et al. were white and all the subjects in our study were Japanese. The effect of DED on healthrelated QOL may differ with ethnicity. The average age and sex distribution also differed between the two studies. Average age was significantly higher in the study of Mertzanis et al. compared with our study. These differences may have had an effect on health-related QOL for DED in these two studies.

The present study showed an association between DED and QOL in non-clinic-based subjects in Japan. DED adversely affected QOL, especially mental health in males. A similar tendency was observed regardless of sex, and MCS was lower in the DED than Non-DED Group (Table 5). DED should be viewed not only as an eye disorder, but also as a condition that affects mental health in non-clinicbased subjects.

There were several limitations to the present study. First, the study subjects were employees of a single organization and may not be representative of the general Japanese population. Recently, the amount of work performed using VDTs has greatly increased in Japan (Uchino et al. 2013); DED symptoms reported by VDT users include eyestrain, reduced visual acuity, ocular pain, and dry eyes (Thomson 1998). University staff are involved in many VDT operations, therefore, prevalence of DED might be higher than that in the general population (Uchino et al. 2011). Second, we surveyed medical history for hypertension, diabetes, hyperlipidemia, and psychiatric disease but could not find a history of menopausal disorders, hormone therapy or LASIK, and dietary habits, including caffeine and supplement use. Third, differences in consulting behavior for DED by sex should also have been considered.

In this study, we showed that DED was associated with reduced mental-health-related QOL. However, this study was cross-sectional. Therefore, the cause of DED and reduced mental QOL is still unclear. A prospective study that evaluates subjects with DED symptoms and reduced mental-health-related QOL will give us stronger evidence for a causal association. Furthermore, we should establish whether treatment for DED symptoms improves mental-health-related QOL in subjects with DED. If treatment for DED could improve mental-health-related QOL, treatment for DED has the possibility of being a new therapy for improving mental-health-related QOL. 


\section{Conflict of Interest}

The authors declare no conflict of interest.

\section{References}

Fukuhara, S., Bito, S., Green, J., Hsiao, A. \& Kurokawa, K. (1998a) Translation, adaptation, and validation of the SF-36 Health Survey for use in Japan. J. Clin. Epidemiol., 51, 10371044.

Fukuhara, S. \& Suzukamo, Y. (2004) Manual of SF-36v2 Japanese version. Institute for Health Outcomes \& Process Evaluation Research, Kyoto, Japan.

Fukuhara, S., Ware, J.E. Jr., Kosinski, M., Wada, S. \& Gandek, B. (1998b) Psychometric and clinical tests of validity of the Japanese SF-36 Health Survey. J. Clin. Epidemiol., 51, 10451053.

Galor, A., Feuer, W., Lee, D.J., Florez, H., Carter, D., Pouyeh, B., Prunty, W.J. \& Perez, V.L. (2011) Prevalence and risk factors of dry eye syndrome in a United States veterans affairs population. Am. J. Ophthalmol., 152, 377-384.

Gulati, A., Sullivan, R., Buring, J.E., Sullivan, D.A., Dana, R. \& Schaumberg, D.A. (2006) Validation and repeatability of a short questionnaire for dry eye syndrome. Am. J. Ophthalmol., 142, 125-131.

Guo, B., Lu, P., Chen, X., Zhang, W. \& Chen, R. (2010) Prevalence of dry eye disease in Mongolians at high altitude in China: the Henan eye study. Ophthalmic Epidemiol., 17, 234-241.

Kim, K.W., Han, S.B., Han, E.R., Woo, S.J., Lee, J.J., Yoon, J.C. \& Hyon, J.Y. (2011) Association between depression and dry eye disease in an elderly population. Invest. Ophthalmol. Vis. Sci., 52, 7954-7958.

Le, Q., Zhou, X., Ge, L., Wu, L., Hong, J. \& Xu, J. (2012) Impact of dry eye syndrome on vision-related quality of life in a nonclinic-based general population. BMC Ophthalmol., 12, 22.

Lemp, M.A., Baudouin, C., Baum, J., Dogru, M., Foulks, G.N., Kinoshita, S., Laibson, P., McCulley, J., Murube, J., Pflugfelder, S.C., Rolando, M. \& Toda, I. (2007) The definition and classification of dry eye disease. Report of the definition and classification subcommittee of the international dry eye workshop 2007. Ocul. Surf., 5, 75-92.

Li, M., Gong, L., Chapin, W.J. \& Zhu, M. (2012) Assessment of vision-related quality of life in dry eye patients. Invest. Ophthalmol. Vis. Sci., 53, 5722-5727.

McCarty, C.A., Bansal, A.K., Livingston, P.M., Stanislavsky, Y.L.
\& Taylor, H.R. (1998) The epidemiology of dry eye in Melbourne, Australia. Ophthalmology, 105, 1114-1119.

Mertzanis, P., Abetz, L., Rajagopalan, K., Espindle, D., Chalmers, R., Snyder, C., Caffery, B., Edrington, T., Simpson, T., Nelson, J.D. \& Begley, C. (2005) The relative burden of dry eye in patients' lives: comparisons to a U.S. normative sample. Invest. Ophthalmol. Vis. Sci., 46, 46-50.

Miljanovic, B., Dana, R., Sullivan, D.A. \& Schaumberg, D.A. (2007) Impact of dry eye syndrome on vision-related quality of life. Am. J. Ophthalmol., 143, 409-415.

Moss, S.E., Klein, R. \& Klein, B.E. (2008) Long-term incidence of dry eye in an older population. Optom. Vis. Sci., 85, 668-674.

Nichols, K.K., Mitchell, G.L. \& Zadnik, K. (2002) Performance and repeatability of the NEI-VFQ-25 in patients with dry eye. Cornea, 21, 578-583.

Sakane, Y., Yamaguchi, M., Yokoi, N., Uchino, M., Dogru, M., Oishi, T., Ohashi, Y. \& Ohashi, Y. (2013) Development and validation of the Dry Eye-Related Quality-of-life Score questionnaire. JAMA Ophthalmol., 131, 1331-1338.

Schaumberg, D.A., Buring, J.E., Sullivan, D.A. \& Dana, M.R. (2001) Hormone replacement therapy and dry eye syndrome. JAMA, 286, 2114-2119.

Schaumberg, D.A., Sullivan, D.A., Buring, J.E. \& Dana, M.R (2003) Prevalence of dry eye syndrome among US women. Am. J. Ophthalmol., 136, 318-326.

Schein, O.D., Munoz, B., Tielsch, J.M., Bandeen-Roche, K. \& West, S. (1997) Prevalence of dry eye among the elderly. Am. J. Ophthalmol., 124, 723-728.

Thomson, W.D. (1998) Eye problems and visual display terminals: the facts and the fallacies. Ophthalmic. Physiol. Opt., 18, 111-119.

Uchino, M., Nishiwaki, Y., Michikawa, T., Shirakawa, K., Kuwahara, E., Yamada, M., Dogru, M., Schaumberg, D.A., Kawakita, T., Takebayashi, T. \& Tsubota, K. (2011) Prevalence and risk factors of dry eye disease in Japan: Koumi study. Ophthalmology, 118, 2361-2367.

Uchino, M., Yokoi, N., Uchino, Y., Dogru, M., Kawashima, M., Komuro, A., Sonomura, Y., Kato, H., Kinoshita, S., Schaumberg, D.A. \& Tsubota, K. (2013) Prevalence of dry eye disease and its risk factors in visual display terminal users: the Osaka study. Am. J. Ophthalmol., 156, 759-766.

Wang, T.J., Wang, I.J., Hu, C.C. \& Lin, H.C. (2012) Comorbidities of dry eye disease: a nationwide population-based study. Acta Ophthalmol., 90, 663-668. 